\title{
Diagnóstico de Ca. de Cuello *
}

\section{CITOLOGIAS SOSPECHOSAS EN ANALISIS ESTADISTICO DE 97.515 EXAMENES}

\author{
Dr. Gamboa González Alvaro \\ Universidad Industrial de Santander \\ Dr. Cortés Caballero Carlos \\ Patólogo Profamilia, Bucaramanga
}

Grupo Citólogas de Profamilia y

Liga de Lucha contra el Cáncer, Bucaramanga.

\section{INTRODUCCION}

A pesar de no ser la citología vaginal una prueba obsolutamente concluyente del estado del cuello uterino (1) es sin embargo el examen más al alcance no solo de especialistas sino del médico general y personal paramédico, que actua en zonas alejadas, donde debe practicarse, a toda mujer sexualmente activa, independiente de su estado y edad, por el riesgo de desarrollar carcinoma cervical o displasia. (2-3). Sí tenemos en cuenta que en Colombia la población femenina en edad fecunda es aproximadamente un $250 / 0$, en éste momento tendríamos un volúmen de 5 millones de mujeres que deberían ser objeto de análisis citológicos anuales. Sin embargo hemos podido observar, que la población que está gozando de éste beneficio, en Bucaramanga, por ejemplo es solo aquella que acude en busca de un método de control familiar ya que obligatoriamente se les somete a éste examen, no así a la población que busca atención hospitalaria donde éste procedimiento no es de rutina. Por último debemos descartar la población rural, la cual tampoco tiene acceso a éstos servicios; un $30 \% / 0$, que lo forman aproximadamente 1500.000 mujeres colombianas.

Lógicamente la actividad epidemiológica está orientada en nuestro medio al estu-

* Trabajo presentado en el XII Congreso Colombiano de Obstetricia y Ginecología de Medellín Diciembre de 1.977. dio de enfermedades infecciosas por el gran impacto que tienen en nuestra morbi-mortalidad; sin embargo no debemos olvidar que éste rubro es controlable fácilmente con la aplicación de medidas de salud pública y que el cáncer va adquiriendo importancia a medida que aquellas disminuyen. Esto es de fácil comprobación histórica, pues se ha observado que con el despeque de un país hacia el desarrollo, las enfermedades transmisibles que ocupaban una gran frecuencia, pasan a un lugar secundario, (2o/o) y tanto el cáncer como las enfermedades cardiovascualres aparecen en primer lugar con un 75o/o del grupo total como causa de muerte (4).

Es de esperar que con las estrategias que el Gobierno ha programado dentro del plan Nacional de Salud se dé cumplimiento a las actividades en cada uno de los niveles y especialmente los que hacen relación a la toma y procedimiento de citología vaginal y de cervix y con más énfasis a nivel rural y local.

\section{PROCEDIMIENTO}

Se han acumulado 67.879 citologías de mujeres atendidas en los centros de Planificación Familiar de Bucaramanga y Cúcuta y se han comparado con 29.636 estudiadas en la Liga de Lucha Contra el Cáncer de Bucaramanga en los años 1971 a 1977 formando un gran total de 97.515. Esta labor es la continuación de un estudio iniciado en 1963 y que hoy actualizamos (5-6). Las 
Tabla I

FRECUENCIA DE CITOLOGIAS SOSPECHOSAS EN BUCARAMANGA Y OTROS SITIOS

\begin{tabular}{lllllll} 
AÑO & \multicolumn{2}{l}{ PRO-B/GA. } & \multicolumn{2}{l}{ LIGA B/GA. } & \multicolumn{2}{l}{ PRO-CUCUTA } \\
& Total & Tasa & Total & Tasa & \multicolumn{2}{c}{ Total } \\
& & & & & & \\
1.969 & & & 2.033 & 1.7 & & \\
1.970 & & & 1.775 & 6.76 & & \\
1.971 & 5.890 & 1.32 & 1.414 & 1.27 & 3.008 & 0.83 \\
1.972 & 7.583 & 0.99 & 2.222 & 2.07 & 3.815 & 0.58 \\
1.973 & 7.963 & 0.69 & 2.870 & 2.37 & 3.998 & 0.50 \\
1.974 & 7.854 & 0.80 & 3.640 & 3.54 & 2.228 & 0.72 \\
1.975 & 5.595 & 0.79 & 3.737 & 2.41 & 1.569 & 1.59 \\
1.976 & 6.661 & 0.70 & 5.158 & 1.80 & 2.441 & 0.77 \\
1977 & 6.134 & 0.58 & 6.787 & 3.52 & 3.140 & 0.44
\end{tabular}

TOTAL

citologías se han agrupado siguiendo la clasificación original de Papanicolau (7).

\section{ANALISIS (Tabla I y Gráfica)}

Detalles muy importantes debemos advertir en los dos últimos años respecto al porcentaje de citologías sospechosas. Como lo habíamos previsto, la tendencia en Profamilia de Bucaramanga es a permanecer estable o quizá disminuir en una forma muy leve, en contraposición a lo ocurrido en la LIga en donde a través de los años el comportamiento ha sido muy irregular, no siendo posible predecir que va a ocurrir y cuál sea la causa del aumento acelerado en el último año. Como consecuencia de lo anterior de 40 citologías sospechosas para el año de 1976 pasamos a un volúmen de 84 en 1977 para Bucaramanga (Liga y Profamilia). Este cambio llama la atención hacia algo raro que pueda estar ocurriendo en la Liga en los dos últimos años ya que el volúmen de exámenes sospechosos pasó de 96 a 239 o sea un aumento en un $150 \%$ /o aproximadamente y la frecuencia de las sospechosas pasó de 1.8 a 3.52o/o llegando a niveles parecidos a los de 1974. Puede ser que la población que allí asiste últimamente sea diferente en su estructura, como por ejemplo mayor edad, alta paridad y mujeres con un más alto indice de relaciones sexuales, o que se esten remitiendo pacientes en los cuales hay alguna sospecha de Ca. y por tanto llevan una gran probabilidad de quedar incluídas en dicho grupo. Además, pueden incidir otros factores de técnica y de interpretación.

Los resultados de Profamilia en ambas ciudades son consecuentes con los objetivos similares que persiguen éstas Instituciones y por ello vemos la tendencia a presentar resultados similares en cuanto a cifras de exámenes sospechosos.

En 1977 en Bucaramanga, de cada 100 citologías practicadas 2.15 fueron sospechosas (278 sospechosas /12.921) o sea 84 sospechosas por cada 100.000 habitantes (278 ( 330.000) estimando que la población de Bucaramanga para 1977 es de 330.000 habitantes. (En la gráfica no se aprecia una tendencia a disminuir el riesgo de Ca. de Cuello; y en la Liga por el contrario aumenta el número de casos en forma acelerada. 
FRECUENCIAS DE CITOLOGIAS SOSPECHOSAS EN BUCARAMANGA

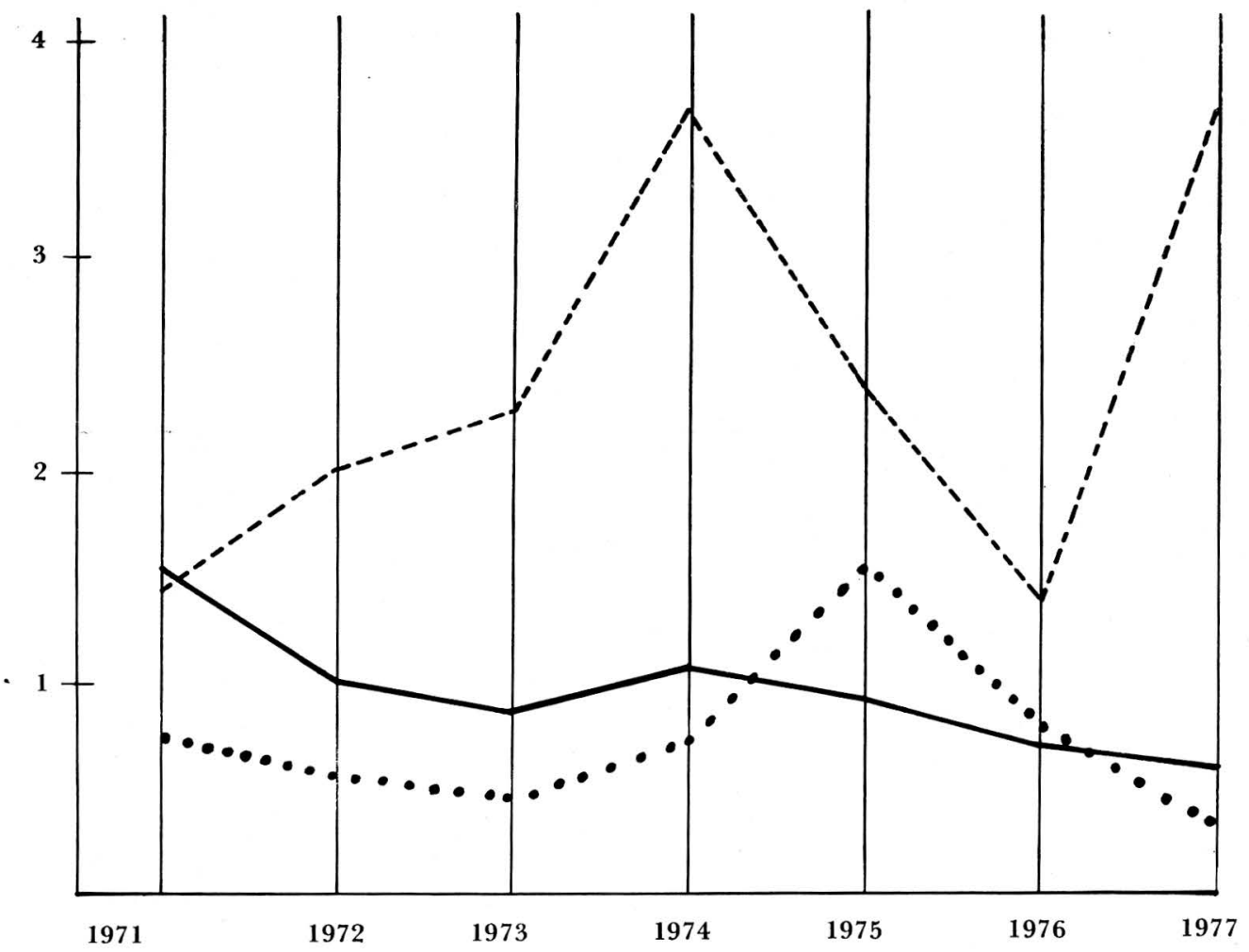

Liga Bucaramanga

Profamilia Bucaramanga

Profamilia Cúcuta

\section{CONCLUSIONES}

1. En Bucaramanga el $2.150 / 0$ de las citologías son sospechosas. de Ca. de cuello.

2. El comportamiento de los exámenes efectuados en la Liga Contra el Cáncer es muy diferente al de Profamilia, quizá obedeciendo a diferencias en la estructura poblacional y teniendo en cuenta los diferentes objetivos.

3. El riesgo de las pacientes sospechosas no tiende a disminuir y por el contrario trata de aumentar.

4. Es un problema de Salud Pública que merece la atención de las autoridades sanitarias.

\section{RESUMEN}

Se analizan 97.515 estudios de colpocitología, verificados en 67.879 mujeres atendidas en los Centros de Planificación Familiar de las ciudades de Bucaramanga y Cúcuta, y 29.636 practicados por la Liga Contra el Cáncer de Bucaramanga entre 1971 y 1977

Los resultados obtenidos en el análisis heche de acuerdo con la clasificación de $\mathrm{Pa}$ panicolau, muestran un notable aumento de casos sospechosos de malignidad hasta 1974, seguido de descenso y nuevo aumento en las estadísticas de la Liga Contra el Cáncer de Bucaramanga. En contraste, la casuística de Profamilia, especialmente en 
Bucaramanga, muestra un notable descenso en la frecuencia de las citologías sopechosas.

Se consideran la importancia y las posibles causas de aumento en la frecuencia de Pap III, en el primer grupo estudiado.

\section{SUMMARY}

97.515 studies of colpocitology are been analized. These studies were made on 67 . 879 women treated by the Centers of $\mathrm{Fa}$ mily Planning of the cities of Bucaramanga and Cúcuta, and 29.636 thar were practiced by the Liga Contra el Cancer Bucaramanga, from 1971 to 1977 .

The results obtained in the analysis done in agreement with the Papanicolau classification, shows a noticiable increase in the number of suspecious melignity cases up to 1974. This increase was followed by a decrease and later by a new increase in the statistics of the Liga Contra el Cáncer of Bucaramanga. In contrast, the casuistics of Profamilia, specially in Bucaramanga, shows a noticeable decrease in franquency of suspicious citologies.

The importance and the possible causes of the increase of frequency of Pap III in the first studied group, are considered.

\section{BIBLIOGRAFIA}

1. Stafl Adolf and Co. Clinical Obstetric and Gynecology Vol. 16 No. 2 1976.
2. Snyder Richard N. M. M. and Co. Dysplasia and Carcinoma in situ of the uterine cervix.

Am J Obstet Gynecol 124-7- 751-55 April 1-1976.

3. Bueno Miguel - Detección precoz de cancer del cuello uterino. Primer Simposio Latinoamericano de detección precoz de enfermedad.

4. Rincón Manuel de Hollain Alejandro Fepafeum Mortalidad segundo tomo Pág. 175.

5. Gamboa G.A., Cortés C.C. y Col. Tendencia al Cancer Genital en Bucaramanga T. M. Colombia No. 601 LT-9 A 15-18. Mayo 6 - 1975.

6. Gamboa G. A., Cortés C.C. y Col. Tendencia al Cancer Genital en Bucaramanga. T. M. Colombia No. 631 LIV No. 3 Pag. 36-37 Agosto de 1976.

7. Papanicolau George N. Altas of Exfoliative Citology pag. 21 Harward University Press. Cambridge, Mass 1963.

8. Estudio apidermiológico de Cancer del Cervix 1974. Ministerio de Salud Pública - Colombia.

9. Jubsawall D. J. et al Assesment of Dirsk Patterms in cancer of the Cervix Cancer 7; 250 a 268 - 1971.

10. Masubuchik et al Epidemiology Stuches on uterine Cancer at Cancer Institu te Hospital Tokio. Japan 1972.

11. Maland Citado (8) Pág. 25. 